\title{
Numerical Solution of the Riemann Problem for Two-Fimensional Gas Dynamics
}

\section{Report}

\section{Author(s):}

Schulz-Rinne, Carsten Werner; Collins, James P.; Glaz, Harland M.

Publication date:

1992-03

Permanent link:

https://doi.org/10.3929/ethz-a-004283302

Rights / license:

In Copyright - Non-Commercial Use Permitted

Originally published in:

SAM Research Report 1992-02 


\title{
Numerical Solution of the Riemann Problem for Two-Dimensional Gas Dynamics
}

\author{
Carsten W. Schulz-Rinne, \\ James P. Collins ${ }^{1}$ and Harland M. Glaz ${ }^{2}$
}

Research Report No. 92-02

March 1992

Seminar für Angewandte Mathematik

Eidgenössische Technische Hochschule

CH-8092 Zürich

Switzerland

\footnotetext{
${ }^{1}$ Information and Mathematical Sciences Branch, Naval Surface Warfare Center, White Oak, Silver Spring, Maryland 20903

2 Department of Mathematics, University of Maryland, College Park, Maryland 20742
} 


\title{
Numerical Solution of the Riemann Problem for Two-Dimensional Gas Dynamics
}

\author{
Carsten W. Schulz-Rinne, James P. Collins ${ }^{1}$ and Harland M. Glaz ${ }^{2}$
}

Seminar für Angewandte Mathematik

Eidgenössische Technische Hochschule

CH-8092 Zürich

Switzerland

Research Report No. 92-02 March 1992

\begin{abstract}
The Riemann problem for two-dimensional gas dynamics with isentropic and polytropic gas is considered. The initial data is constant in each quadrant and chosen so that only a rarefaction wave, shock wave or slip line connects two neighboring constant initial states. With this restriction sixteen (resp. fifteen) genuinely different wave combinations for isentropic (resp. polytropic) gas exist. For each configuration the numerical solution is analyzed and illustrated by contour plots. Additionally, the required relations for the initial data and the symmetry properties of the solutions are given. The chosen calculations correspond closely to the cases studied by T. Zhang and Y. Zheng, SIAM J. Math. Anal. 21 (1990), 593-630, so that the analytical theory can be directly compared to our numerical study.
\end{abstract}

Keywords: Riemann problem, gas dynamics, Godunov method, wave interaction AMS(MOS) Subject Classification: 35A40, 35L65, 35L67, 65M06, 76N15

\footnotetext{
${ }^{1}$ Information and Mathematical Sciences Branch, Naval Surface Warfare Center, White Oak, Silver Spring, Maryland 20903

${ }^{2}$ Department of Mathematics, University of Maryland, College Park, Maryland 20742
} 


\section{IntroduCtion}

The interaction of elementary waves for systems of hyperbolic conservation laws is the key mechanism in determining the qualitative properties of solutions to more general initial value problems. For the genuinely nonlinear case and one space dimension, these basic interactions along with the Riemann problem can be worked out analytically at least for small enough jumps in wave strength [15] and are often solvable for large jumps as well [3], [17]. This knowledge is the basic building block in using techniques such as the random choice method ( $\mathrm{RCM})$ to obtain partial existence results [8] as well as information about the qualitative behavior of solutions [12], and this is especially the case for certain systems which are derived from physical principles, e.g., gas dynamics. Indeed, one can go further and conjecture that RCM with enough mesh points and sufficient accuracy in the Riemann problem solution will almost always produce a very close approximate to the exact solution for scalar conservation laws, gas dynamics and certain other systems in the $1 \mathrm{D}$ planar case.

The idea of using elementary waves and Riemann problems as building blocks in constructing solutions, being so successful in $1 \mathrm{D}$, is natural to attempt in $2 \mathrm{D}$ (and $3 \mathrm{D}$ ). For the scalar case, a great deal of work has been done [10], [11], [16], [18] and much is known about the analytic structure of solutions. However, even here it seems difficult to work in analogy to the methods of [8], [12] and obtain deep results for general data; also, finite difference schemes based directly on 2D Riemann problem solutions have not appeared.

For gas dynamics and two space dimensions, the situation can be expected to be quite complex and is certainly difficult - very few analytic results are available. Our approach here is to use a high resolution finite difference scheme as an experimental tool and try to catalogue the phenomenology of the $2 \mathrm{D}$ gas dynamic Riemann problem for a restricted set of initial data; our choices have been strongly influenced by the conjectures reported in [19]. A similar approach using the front tracking method is also possible [9], but our results here indicate that such an effort would be awkward to implement. However, the set of results obtained here, suitably augmented to account for a larger data set, might be useful in constructing numerical methods along this line by supplying the appropriate jump conditions at solution singularities.

The most important feature of elementary waves and Riemann problems is that their solutions may be assumed to be self-similar; this reduces the number of dimensions by one and this leads to the analytic theory for the 1D case. The dimensional reduction in $2 \mathrm{D}$ is from three to two dimensions and the resulting system is still a PDE. A set of interesting self-similar data for gas dynamics, from an historical point of view, is the problem of oblique shock wave reflection which can be experimentally simulated by situating a wedge in a shock tube downstream of the incident shock. Experimental, analytic and numerical investigations of this problem go back to E. Mach and J. von Neumann; the extensive literature is discussed in [5]. The numerical scheme used to obtain the results in our work here has been implemented for this configuration as well [6], [7]. It has been conjectured [19] and will be shown here that at least some of these results also arise from $2 \mathrm{D}$ Riemann problems.

The collection of self-similar data in $2 \mathrm{D}$ is very diverse, as already illustrated by the possible wedge configurations and their solutions. Actually, any collection of rays centered at some point (always the origin here) and separated by constant states will 
lead to a self-similar solution. Our study here is restricted to the special case of four constant states, one in each quadrant. We further restrict by insisting that the waves separating each pair of adjoining states be a single elementary wave; this corresponds to the set of conjectures proposed in [19] which partially inspired the work here.

We remark that even the case of a single planar shock wave situated in $\mathbb{R}^{2}$ is not necessarily trivial, at least numerically. Indeed, certain conditions must be satisfied by the data and the equation of state in order that the wave be stable [13]. However, stability is guaranteed for the genuinely nonlinear case and our work so far has been for polytropic gases.

All computations are performed using the second order Eulerian Godunov method as formulated in [1] along with the dissipative mechanisms discussed in [2]. The capabilities of this scheme applied to a wide variety of applications are summarized in [4]. The boundary conditions are trivial for this study, of course. The initial data is laid down on the grid with the jumps at (the Cartesian) grid interfaces. The calculations are started smoothly by taking the initial CFL constraint to be very severe and gradually relaxing it. The $(x, y)$-plane is covered with a uniform mesh containing 400 cells in each direction for all problems reported here. The calculations were performed on Sun workstations at the ETH Zurich.

The equations of motion, the Riemann problem initial data and the resulting selfsimilar equations are discussed in Section 2; this section also contains a summary of the classification based on [19] and derived in [14].

In Section 3 the numerical solutions and their structures are presented and analysed. Through observation of the time dependent solution, it is clear that our mesh is sufficient for the solutions to relax to their pseudosteady states; thus, our illustrations may be assumed to be situated in the $(\xi, \eta)$-plane. A few computations exhibit an unsteady Kelvin-Helmholtz instability across slip surfaces. For these cases, one cannot assume the existence of a true pseudosteady state although the overall structures are stable in time; see [5] for further discussion. The results are illustrated by contour plots of the density and self-similar Mach number. In the $(\xi, \eta)$-plane the pseudostationary flow is transonic. The four elementary waves, extending from the boundary of the domain parallel to the coordinate axes, interact and/or reach the region of subsonic flow. In the cases with four shock waves the interaction of the shock fronts results in simple, complex or double Mach reflections depending on the initial strength of the waves. Four slip lines lead to large-scale vortex creation in one case. With some wave combinations the region of subsonic flow is partly bounded by newly created shocks. Our conclusions are presented in Section 4.

\section{Problem formulation and Classification}

The Riemann problem for gas dynamics in two space dimensions, both for isentropic and polytropic gas, is formulated. We start with the Euler equations of inviscid compressible isentropic flow consisting of the equations for conservation of mass and momentum. For polytropic gas we have an additional equation for the conservation of energy. The conservation form of these equations in Cartesian coordinates is

$$
U_{t}+F(U)_{x}+G(U)_{y}=0
$$


where

$$
U=\left(\begin{array}{c}
\rho \\
\rho u \\
\rho v
\end{array}\right), \quad F(U)=\left(\begin{array}{c}
\rho u \\
\rho u^{2}+p \\
\rho u v
\end{array}\right), \quad G(U)=\left(\begin{array}{c}
\rho v \\
\rho u v \\
\rho v^{2}+p
\end{array}\right)
$$

for isentropic gas and

$$
U=\left(\begin{array}{c}
\rho \\
\rho u \\
\rho v \\
\rho E
\end{array}\right), \quad F(U)=\left(\begin{array}{c}
\rho u \\
\rho u^{2}+p \\
\rho u v \\
u(\rho E+p)
\end{array}\right), \quad G(U)=\left(\begin{array}{c}
\rho v \\
\rho u v \\
\rho v^{2}+p \\
v(\rho E+p)
\end{array}\right)
$$

for polytropic gas. Here $\rho$ is the density, $u$ the $x$-velocity component, $v$ the $y$-velocity component, $p$ the pressure, and $E$ the total specific energy. The system is closed by specifying an equation of state. For isentropic gas we take $p=A \rho^{\gamma}$ where $A>0$ and $\gamma>1$ are constants. For polytropic gas we have instead

$$
E=\frac{1}{(\gamma-1)} \frac{p}{\rho}+\frac{u^{2}+v^{2}}{2}
$$

where $\gamma>1$ is constant. The number $\gamma$ is called the polytropic exponent and, since it is assumed constant in the present study, may be interpreted physically as the ratio of specific heats for the polytropic case.

The characteristic speeds of (1) in the $x$ - (or $y$-) direction, i. e., the eigenvalues of the Jacobian matrix $\nabla_{U} F$ (or $\nabla_{U} G$ ) are $\lambda_{-}=u-c, \lambda_{0}=u$ and $\lambda_{+}=u+c$ (or $\lambda_{-}=v-c$, $\lambda_{0}=v$ and $\left.\lambda_{+}=v+c\right)$. Here the sound speed $c$ is defined by $c^{2}=\gamma p / \rho$.

The Riemann problem in the $(x, y)$-plane is the initial value problem for (1) with initial data

$$
(\rho, u, v)(x, y, 0)=\left(\rho_{i}, u_{i}, v_{i}\right), \quad i=1, \ldots, 4
$$

for isentropic gas and

$$
(p, \rho, u, v)(x, y, 0)=\left(p_{i}, \rho_{i}, u_{i}, v_{i}\right), \quad i=1, \ldots, 4
$$

for polytropic gas where $i$ denotes the $i$ th quadrant.

The solution is a function of the similarity variables $\xi=x / t$ and $\eta=y / t$. In these new variables (1) becomes

$$
-\xi U_{\xi}-\eta U_{\eta}+F(U)_{\xi}+G(U)_{\eta}=0 .
$$

Introducing the so-called pseudovelocities $\tilde{u}=u-\xi$ and $\tilde{v}=v-\eta$, the system (3) is equivalent to

$$
F(\tilde{U})_{\xi}+G(\tilde{U})_{\eta}+S(\tilde{U})=0
$$

where

$$
\tilde{U}=\left(\begin{array}{c}
\rho \\
\rho \tilde{u} \\
\rho \tilde{v}
\end{array}\right), \quad S(\tilde{U})=\left(\begin{array}{c}
2 \rho \\
3 \rho \tilde{u} \\
3 \rho \tilde{v}
\end{array}\right)
$$


for isentropic gas and

$$
\tilde{U}=\left(\begin{array}{c}
\rho \\
\rho \tilde{u} \\
\rho \tilde{v} \\
\rho \tilde{E}
\end{array}\right), \quad S(\tilde{U})=\left(\begin{array}{c}
2 \rho \\
3 \rho \tilde{u} \\
3 \rho \tilde{v} \\
2(\rho \tilde{E}+p)+\rho\left(\tilde{u}^{2}+\tilde{v}^{2}\right)
\end{array}\right)
$$

with

$$
\tilde{E}=\frac{1}{(\gamma-1)} \frac{p}{\rho}+\frac{\tilde{u}^{2}+\tilde{v}^{2}}{2}
$$

for polytropic gas. The system (4) can be expressed in quasilinear form as

$$
V_{\xi}+A V_{\eta}+B=0
$$

where

$$
A=\left[\frac{\partial F}{\partial V}\right]^{-1}\left[\frac{\partial G}{\partial V}\right], \quad B=\left[\frac{\partial F}{\partial V}\right]^{-1} S
$$

and $V$ is an arbitrary set of characteristic coordinates such that $\operatorname{det}(A) \neq 0$. Then the eigenvalues of $A$ are

$$
\tilde{\lambda}_{0}=\frac{\tilde{v}}{\tilde{u}} \quad \text { and } \quad \tilde{\lambda}_{ \pm}=\frac{\tilde{u} \tilde{v} \pm c \sqrt{\tilde{u}^{2}+\tilde{v}^{2}-c^{2}}}{\tilde{u}^{2}-c^{2}}=\frac{\tilde{v}^{2}-c^{2}}{\tilde{u} \tilde{v} \mp c \sqrt{\tilde{u}^{2}+\tilde{v}^{2}-c^{2}}}
$$

The initial value problem becomes a boundary value problem at infinity:

$$
\left.\begin{array}{rl}
(\rho, u, v)(\xi, \eta) & \rightarrow\left(\rho_{i}, u_{i}, v_{i}\right) \\
& \text { or } \\
(p, \rho, u, v)(\xi, \eta) & \rightarrow\left(p_{i}, \rho_{i}, u_{i}, v_{i}\right)
\end{array}\right\} \text { for } \xi^{2}+\eta^{2} \rightarrow \infty \text { and }\left\{\begin{array}{l}
\xi>0, \eta>0, i=1 \\
\xi<0, \eta>0, i=2 \\
\xi<0, \eta<0, i=3 \\
\xi>0, \eta<0, i=4
\end{array}\right.
$$

The self-similar solution in the $(\xi, \eta)$-plane is called pseudostationary flow. Far enough away from the origin the general solution consists of four planar waves, each parallel to one of the coordinate axes, separating the four constant initial states; also, the eigenvalues $\tilde{\lambda}_{ \pm}$are guaranteed to be real. In general, a planar wave is formed by up to three elementary waves corresponding to the eigenvalues $\lambda_{-}, \lambda_{0}$ and $\lambda_{+}$: a backward rarefaction wave $\overleftarrow{R}$ or shock wave $\overleftarrow{S}$, a slip line (resp. a contact discontinuity) for isentropic (resp. polytropic) gas ${ }^{1} J$, and a forward rarefaction wave $\vec{R}$ or shock wave $\vec{S}$. In our study of the interaction of the four planar waves we restrict ourselves to situations where each planar wave is a single elementary wave.

The Riemann problem is classified according to the combination of the four elementary planar waves used to define it. Thus we assume that the initial data (2) or (2') are chosen so that only a rarefaction wave, shock wave or slip line connects two neighboring constant states. Under this assumption it was proved in [14] that sixteen (resp. fifteen) different

\footnotetext{
${ }^{1}$ In the following the use of the term slip line always denotes a slip line for isentropic gas but should be read to include the possibility of a contact discontinuity for polytropic gas.
} 
configurations for the Riemann problem for isentropic (resp. polytropic) gas in two space dimensions exist (symmetric or rotated configurations are systematically eliminated):

$\begin{array}{llll}4 R: & \vec{R}_{21} \vec{R}_{32} \vec{R}_{34} \vec{R}_{41} & \vec{R}_{21} \overleftarrow{R}_{32} \overleftarrow{R}_{34} \vec{R}_{41} \\ 4 S: & \overleftarrow{S}_{21} \overleftarrow{S}_{32} \overleftarrow{S}_{34} \overleftarrow{S}_{41} & \overleftarrow{S}_{21} \vec{S}_{32} \vec{S}_{34} \overleftarrow{S}_{41} & \\ 2 R+2 S: & & \vec{R}_{21} \vec{S}_{32} \overleftarrow{R}_{34} \overleftarrow{S}_{41} & \text { (only for isentropic gas) } \\ 4 J: & \overleftarrow{J}_{21} J_{32} J_{34} J_{41} & & J_{21} J_{32} J_{34} J_{41} \\ 2 J+2 R: & \vec{R}_{21} J_{32} J_{34} \vec{R}_{41} & \overleftarrow{R}_{21} J_{32} J_{34} \overleftarrow{R}_{41} & J_{21} \vec{R}_{32} J_{34} \vec{R}_{41} \\ 2 J+2 S: & \overleftarrow{S}_{21} J_{32} J_{34} \overleftarrow{S}_{41} & \vec{S}_{21} J_{32} J_{34} \vec{S}_{41} & J_{21} \overleftarrow{S}_{32} J_{34} \overleftarrow{S}_{41} \\ 2 J+R+S: & \vec{R}_{21} J_{32} J_{34} \overleftarrow{S}_{41} & \overleftarrow{R}_{21} J_{32} J_{34} \vec{S}_{41} & J_{21} \overleftarrow{S}_{32} J_{34} \vec{R}_{41}\end{array}$

In this table and in the following, $E_{i j}$ with $E \in\{J, \overleftarrow{R}, \vec{R}, \overleftarrow{S}, \vec{S}\}$ and $i, j \in\{1,2,3,4\}$ denotes an elementary wave $E$ between the $i$ th and $j$ th quadrant.

\section{Computational results}

In this section we present the numerical solutions to the configurations one by one. For each of them we give the relations which have to be satisfied by the initial data and the symmetry properties of the solution. The formulas for one-dimensional elementary waves between two constant states, which follow from the simple wave and RankineHugoniot relations [3], are stated in [14]. We use the formulas and the abbreviations introduced there; that is, for a given left and right state (denoted by the indices $l$ and $r$ ) we define

$$
\Phi_{l r}:=\frac{2 \sqrt{\gamma}}{\gamma-1}\left(\sqrt{\frac{p_{l}}{\rho_{l}}}-\sqrt{\frac{p_{r}}{\rho_{r}}}\right), \quad \Psi_{l r}^{2}:=\frac{\left(p_{l}-p_{r}\right)\left(\rho_{l}-\rho_{r}\right)}{\rho_{l} \rho_{r}} \quad\left(\Psi_{l r}>0\right)
$$

and

$$
\Pi_{l r}:=\left(\frac{p_{l}}{p_{r}}+\frac{(\gamma-1)}{(\gamma+1)}\right) /\left(1+\frac{(\gamma-1)}{(\gamma+1)} \frac{p_{l}}{p_{r}}\right) .
$$

Furthermore, we describe the main features of the solution in the $(\xi, \eta)$-plane. It always contains a subsonic area $(\tilde{M}<1)$ which is separated from the supersonic area $(\tilde{M}>1)$ by the sonic curve $(\tilde{M}=1)$. Here the self-similar Mach number $\tilde{M}$ is defined by $\tilde{M}^{2}=\left(\tilde{u}^{2}+\tilde{v}^{2}\right) / c^{2}$. In the supersonic domain the curves given by $d \eta / d \xi=\tilde{\lambda}_{ \pm}$are called the $\lambda_{ \pm}$characteristic lines. As in [19] we outline the behavior of these lines inside the rarefaction waves. The numerical solutions are illustrated by contour plots of the density and self-similar Mach number in the $(\xi, \eta)$-plane. In the Mach number plots the subsonic contour lines are dashed. For some configurations containing rarefaction waves the $\lambda_{+(-)}$characteristic lines inside the fan are shown as solid (dashed) lines. The initial data listed in the figure captions, together with the relations given for the corresponding configuration, are always sufficient to uniquely define the solution. 


\section{Cases not involving slip line initial data.}

\section{Four rarefaction waves.}

In this case the flow is isentropic.

Configuration 1: $\quad \vec{R}_{21} \vec{R}_{32} \vec{R}_{34} \vec{R}_{41} \quad$ (Figures 1, 3a)

We have

$$
p_{1}>p_{2}, p_{4}>p_{3}
$$

and

$$
\begin{array}{llll}
u_{2}-u_{1}=\Phi_{21}, & u_{3}-u_{4}=\Phi_{34}, & u_{3}=u_{2}, & u_{4}=u_{1}, \\
v_{4}-v_{1}=\Phi_{41}, & v_{3}-v_{2}=\Phi_{32}, & v_{2}=v_{1}, & v_{3}=v_{4} .
\end{array}
$$

This gives the so-called compatibility condition $\Phi_{21}=\Phi_{34}$. For polytropic gas we have to include the following equations:

$$
\rho_{i} / \rho_{j}=\left(p_{i} / p_{j}\right)^{1 / \gamma} \quad \text { for } \quad(i, j) \in\{(2,1),(3,4),(3,2),(4,1)\}
$$

For the case of a vanishing pressure jump in the $\eta$-direction the sonic curve consists of a circle behind the rarefaction fan and a straight line called the sonic stem extending horizontally from the circle to the front of the wave. Inside the rarefaction fan the $\lambda_{ \pm}$ characteristic lines are parallel to the contour lines between the boundary and the sonic stem. There the lines turn downwind to end at the sonic circle.

For the general case the sonic circles of $\vec{R}_{32}$ and $\vec{R}_{34}$ jointly form the subsonic area behind the interaction of the waves. The rarefaction waves $\vec{R}_{21}$ and $\vec{R}_{41}$ meet at a point $P$ in the first quadrant. The $\lambda_{-}$characteristic through $P$ forms the lower boundary of the undisturbed $\vec{R}_{21}$ wave. It crosses $\vec{R}_{21}$, continues in the second quadrant, turns downward, crosses $\vec{R}_{32}$, enters the third quadrant and ends at the sonic curve. Analogously, the $\lambda_{+}$characteristic through $P$ forms the left boundary of the undisturbed $\vec{R}_{41}$ wave. It crosses $\vec{R}_{41}$, continues in the fourth quadrant, turns left, crosses $\vec{R}_{34}$ and ends at the sonic curve in the third quadrant. The $\lambda_{-}$characteristic lines inside $\vec{R}_{34}$ are parallel to the contour lines inside the fan and turn downwind to end at the sonic curve. The equivalent is true for the $\lambda_{+}$characteristic inside $\vec{R}_{32}$. The traces of the sonic stems in $\vec{R}_{32}$ and $\vec{R}_{34}$ are easily found in the Mach number plot (Fig. 1).

For an extensive range of initial data we found that an intersection of the $\lambda_{ \pm}$characteristic lines at the lower left side of the subsonic area does not occur, compare $[19$, Figs. 4.2 and 4.3].

Configuration 2: $\quad \vec{R}_{21} \overleftarrow{R}_{32} \overleftarrow{R}_{34} \vec{R}_{41} \quad$ (Figures 2, 3b)

We have

$$
p_{1}>p_{2}, p_{4}<p_{3}
$$

and

$$
\begin{array}{llll}
u_{2}-u_{1}=\Phi_{21}, & u_{4}-u_{3}=\Phi_{34}, & u_{3}=u_{2}, & u_{4}=u_{1}, \\
v_{4}-v_{1}=\Phi_{41}, & v_{2}-v_{3}=\Phi_{32}, & v_{2}=v_{1}, & v_{3}=v_{4}
\end{array}
$$

so that the compatibility conditions are $\Phi_{21}=-\Phi_{34}$ and $\Phi_{41}=-\Phi_{32}$. For polytropic gas we include the same additional equations as in Configuration 1. 
Thus we must have $p_{1}=p_{3}$ and $p_{2}=p_{4}$ implying $u_{1}-u_{2}=v_{1}-v_{4}$ and $u_{4}-u_{3}=$ $v_{2}-v_{3}$. Consequently the solutions are symmetric to $\eta-\xi=v_{1}-u_{1}$ and $\xi+\eta=u_{3}+v_{3}$. Therefore the description focuses on the region $\xi+\eta>u_{3}+v_{3}$ of the domain.

The nonconvex subsonic area lies between the four rarefaction waves. On the segment of the sonic curve facing the first quadrant a shock wave appears. The rarefaction waves $\vec{R}_{21}$ and $\vec{R}_{41}$ meet at a point $P$ in the first quadrant. The $\lambda_{-}$characteristic through $P$ forms the lower boundary of the undisturbed $\vec{R}_{21}$ wave. It crosses $\vec{R}_{21}$ and turns slightly downward ending at the sonic curve. Analogously, the $\lambda_{+}$characteristic through $P$ forms the left boundary of the undisturbed $\vec{R}_{41}$ wave. It crosses $\vec{R}_{41}$ and turns slightly left ending at the sonic curve. The other $\lambda_{ \pm}$characteristic lines inside the fans are almost parallel to the corresponding characteristics mentioned above.

The overall structure of our solution corresponds reasonably well with the prediction of [19, Fig. 4.5], although the computed shock on the sonic curve is not present in the prediction. Also, for the calculation presented here as well as for many others performed by us, the $\lambda_{ \pm}$characteristic structure never exhibited tangential incidence with the sonic curve and the behavior depicted in [19, Fig. 4.6] was not observed.

Four shock waves.

Configuration 3: $\overleftarrow{S}_{21} \overleftarrow{S}_{32} \overleftarrow{S}_{34} \overleftarrow{S}_{41} \quad$ (Figures 4, 5)

We have

$$
p_{1}>p_{2}, p_{4}>p_{3}
$$

and

$$
\begin{array}{llll}
u_{2}-u_{1}=\Psi_{21}, & u_{3}-u_{4}=\Psi_{34}, & u_{3}=u_{2}, & u_{4}=u_{1}, \\
v_{4}-v_{1}=\Psi_{41}, & v_{3}-v_{2}=\Psi_{32}, & v_{2}=v_{1}, & v_{3}=v_{4} .
\end{array}
$$

This gives the compatibility conditions $\Psi_{21}=\Psi_{34}$ and $\Psi_{41}=\Psi_{32}$. For polytropic gas the following equations are added:

$$
\rho_{i} / \rho_{j}=\Pi_{i j} \quad \text { for } \quad(i, j) \in\{(2,1),(3,4),(3,2),(4,1)\}
$$

It has been proved in [14] that the pressure inequalities required for this configuration and the compatibility conditions listed above can only be satisfied if $p_{4}=p_{2}$ assuming that $1<\gamma \leqslant 3$ for polytropic gas. Therefore we have to choose $p_{4}=p_{2}$ (which implies $\left.\rho_{4}=\rho_{2}\right)$ and we get $u_{2}-u_{1}=v_{4}-v_{1}$. Hence the solutions are symmetric to $\eta-\xi=v_{1}-u_{1}$ and we can concentrate on the region $\eta-\xi>v_{1}-u_{1}$ of the domain.

For the shock speeds $\sigma_{i j}$ of the shocks $\overleftarrow{S}_{i j}$ the following inequalities hold:

$$
\sigma_{21}<\sigma_{34} \quad \text { and } \quad \sigma_{41}<\sigma_{32}
$$

By definition, they are equivalent to

$$
u_{2}-\sqrt{\frac{\rho_{1}}{\rho_{2}} \frac{\left(p_{1}-p_{2}\right)}{\left(\rho_{1}-\rho_{2}\right)}}<u_{3}-\sqrt{\frac{\rho_{4}}{\rho_{3}} \frac{\left(p_{4}-p_{3}\right)}{\left(\rho_{4}-\rho_{3}\right)}}
$$

and

$$
v_{4}-\sqrt{\frac{\rho_{1}}{\rho_{4}} \frac{\left(p_{1}-p_{4}\right)}{\left(\rho_{1}-\rho_{4}\right)}}<v_{3}-\sqrt{\frac{\rho_{2}}{\rho_{3}} \frac{\left(p_{2}-p_{3}\right)}{\left(\rho_{2}-\rho_{3}\right)}} .
$$


Using $u_{3}=u_{2}, v_{3}=v_{4}$ and the compatibility conditions, both inequalities become

$$
\rho_{1} \rho_{3}<\rho_{2} \rho_{4} \quad \text { which is equivalent to } \quad p_{1} p_{3}<p_{2} p_{4} \text {. }
$$

Considering the symmetry (for a more general argument not using the assumption $p_{2}=$ $p_{4}$, see [19]) we set

$$
p_{1}=r p_{3} \quad \text { and } \quad p_{2}=p_{4}=s p_{3} .
$$

Then we have to prove that

$$
r<s^{2} \quad \text { for } \quad r>s>1
$$

under the compatibility condition

$$
(r-s)\left(s^{-1 / \gamma}-r^{-1 / \gamma}\right)=(s-1)\left(1-s^{-1 / \gamma}\right)
$$

for isentropic gas and

$$
(r-s)^{2} /[(\gamma+1) r+(\gamma-1) s]=(s-1)^{2} /[(\gamma+1)+(\gamma-1) s]
$$

for polytropic gas. The functions

$$
f_{i}(r)=(r-s)\left(s^{-1 / \gamma}-r^{-1 / \gamma}\right)-(s-1)\left(1-s^{-1 / \gamma}\right)
$$

and

$$
f_{p}(r)=(r-s)^{2}[(\gamma+1)+(\gamma-1) s]-(s-1)^{2}[(\gamma+1) r+(\gamma-1) s]
$$

have the following properties:

$$
\begin{aligned}
f_{i}(s) & =-(s-1)\left(1-s^{-1 / \gamma}\right)<0 \\
f_{i}\left(s^{2}\right) & =(s-1)\left(1-s^{-1 / \gamma}\right)\left(s^{(\gamma-1) / \gamma}-1\right)>0 \\
f_{i}^{\prime \prime}(r) & =\gamma^{-2} r^{-(2 \gamma+1) / \gamma}[(\gamma-1) r+(\gamma+1) s]>0 \\
f_{p}(s) & =-2 \gamma s(s-1)^{2}<0 \\
f_{p}\left(s^{2}\right) & =(\gamma-1) s(s-1)^{2}\left(s^{2}-1\right)>0 \\
f_{p}^{\prime \prime}(r) & =2>0
\end{aligned}
$$

Thus the root $r>s$ of $f_{i}$ or $f_{p}$ which is also the solution of the corresponding compatibility condition satisfies $r<s^{2}$.

The shock $\overleftarrow{S}_{21}$ intersects the sonic circle of the constant state in the first quadrant before it meets $\overleftarrow{S}_{32}$ as expected from the shock speed inequalities. $\overleftarrow{S}_{21}$ bends toward the subsonic area. The intersection of $\overleftarrow{S}_{21}$ and $\overleftarrow{S}_{32}$ creates a three-shock configuration. The Mach shock and its symmetric counterpart join and bound the subsonic area. A slip line reaches from the branch point into the subsonic area toward the symmetry 
axis. Depending on the strength of the shocks we observe the different types of Mach reflections: the simple Mach reflection where the area between the reflected shock and the slip line is subsonic, the complex Mach reflection (Fig. 5b) where a part of that area is supersonic, and the double Mach reflection (Figs. 4, 5a) with another threeshock configuration. Thus far, we have not observed regular reflection and for all cases of Mach reflection studied the slip line rolls up into a vortex rather than intersecting the symmetry line at a self-similar stagnation point. The prediction of $[19$, Fig. 5.2] is remarkably accurate. We note that the extra slip lines at the interfaces of the quadrants in Figure 4 are numerical artifacts which have been studied earlier [1], [6] and are due to a 'starting error'.

Configuration 4: $\quad \overleftarrow{S}_{21} \vec{S}_{32} \vec{S}_{34} \overleftarrow{S}_{41} \quad$ (Figure 6)

We have

$$
p_{1}>p_{2}, p_{4}<p_{3}
$$

and the same equations and compatibility conditions as in Configuration 3.

Necessarily we must have $p_{1}=p_{3}$ and $p_{2}=p_{4}$ (which implies $\rho_{1}=\rho_{3}$ and $\rho_{2}=\rho_{4}$ ) yielding $u_{2}-u_{1}=v_{4}-v_{1}$ and $u_{3}-u_{4}=v_{3}-v_{2}$. Consequently the solutions are symmetric to $\eta-\xi=v_{1}-u_{1}$ and $\xi+\eta=u_{3}+v_{3}$. Therefore the description focuses on the region $\eta-\xi>v_{1}-u_{1}$ of the domain.

It is easy to conclude from the equations that $\sigma_{21}<\sigma_{34}$ and $\sigma_{41}<\sigma_{32}$. Accordingly the shock $\overleftarrow{S}_{21}$ interacts with $\vec{S}_{32}$. This interaction creates a pair of three-shock configurations. The subsonic area is bounded by the joining Mach shocks and the reflected shocks so that it has an oval shape. As in Configuration 3 we observe the different types of Mach reflections determined by the strength of the shocks. Illustrated here is a case of simple Mach reflection.

Two rarefaction and two shock waves.

Configuration 6: $\quad \vec{R}_{21} \vec{S}_{32} \overleftarrow{R}_{34} \overleftarrow{S}_{41} \quad$ (Figure 7)

We have

$$
p_{1}>p_{2}, p_{4}<p_{3}
$$

and

$$
\begin{array}{llll}
u_{2}-u_{1}=\Phi_{21}, & u_{4}-u_{3}=\Phi_{34}, & u_{3}=u_{2}, & u_{4}=u_{1}, \\
v_{4}-v_{1}=\Psi_{41}, & v_{3}-v_{2}=\Psi_{32}, & v_{2}=v_{1}, & v_{3}=v_{4},
\end{array}
$$

so that the compatibility conditions are $\Phi_{21}=-\Phi_{34}$ and $\Psi_{41}=\Psi_{32}$.

For polytropic gas the equations

$$
\rho_{3} / \rho_{2}=\Pi_{32}, \quad \rho_{4} / \rho_{1}=\Pi_{41}, \quad \rho_{2} / \rho_{1}=\left(p_{2} / p_{1}\right)^{1 / \gamma} \quad \text { and } \quad \rho_{3} / \rho_{4}=\left(p_{3} / p_{4}\right)^{1 / \gamma}
$$

must be added. As mentioned in Section 2 this configuration is impossible for this case [14].

For isentropic gas the pressure inequalities required for this configuration and the compatibility conditions listed above can only be satisfied if $p_{3}=p_{1}$ and $p_{4}=p_{2}$ [14]. Consequently we have to choose $p_{3}=p_{1}$ and $p_{4}=p_{2}$ (which implies $\rho_{3}=\rho_{1}$ and $\left.\rho_{4}=\rho_{2}\right)$. Thus the solutions are symmetric with respect to the point $(\xi, \eta)=\left(u_{1}, v_{1}\right)$ and we can focus the description on the upper part of the domain. 
The shock $\vec{S}_{32}$ bends downward before it dissolves inside the rarefaction fan of $\vec{R}_{21}$. Thus, the predicted intersection of the shocks $\vec{S}_{32}$ and $\overleftarrow{S}_{41}$ (see [19, Fig. 6.4]) does not occur in our computations; as a consequence, the calculated results do not contain any slip surfaces in contrast to [19, Fig. 6.4]. The rarefaction wave $\overleftarrow{R}_{34}$ turns right in front of the shock $\vec{S}_{32}$. The subsonic area is centered about the point $\left(u_{1}, v_{1}\right)$. The $\lambda_{+}$ characteristic lines inside $\vec{R}_{21}$ are parallel to the contour lines inside the fan and end at the sonic curve.

\section{Cases involving slip line initial data.}

Now we discuss the eleven possible configurations involving slip lines. Besides the relations for the initial data listed for each configuration we have to include additional relations for polytropic gas. For a rarefaction or a shock wave between the $i$ th and $j$ th quadrant $(i, j \in\{1, \ldots, 4\})$ we add

$$
\rho_{i} / \rho_{j}=\left(p_{i} / p_{j}\right)^{1 / \gamma} \quad \text { or } \quad \rho_{i} / \rho_{j}=\Pi_{i j},
$$

respectively.

The predicted results of [19] are considerably more speculative for these more difficult cases. Consequently, a feature by feature comparison with the computed solutions will not be attempted.

\section{Four slip lines.}

Configuration A: $\overleftrightarrow{J_{21} J_{32} J_{34} J_{41}} \quad$ (motion in opposite directions; Figure 8)

We have $p_{1}=p_{2}=p_{3}=p_{4}$ and

$$
u_{1}=u_{2}<u_{3}=u_{4}, \quad v_{1}=v_{4}<v_{3}=v_{2} .
$$

Only for isentropic gas the solutions are symmetric with respect to the point $(\xi, \eta)=$ $\left(\frac{1}{2}\left(u_{1}+u_{3}\right), \frac{1}{2}\left(v_{1}+v_{2}\right)\right)$. The slip lines $J_{21}$ and $J_{32}$ meet the sonic circle of the constant state in the second quadrant and continue as almost straight lines so that a quarter of the sonic circle lies in between. The equivalent is true for the slip lines $J_{34}$ and $J_{41}$ in the fourth quadrant. Inside the subsonic area the slip lines bend and end in spirals. Centered about the point $\left(\frac{1}{2}\left(u_{1}+u_{3}\right), \frac{1}{2}\left(v_{1}+v_{2}\right)\right)$ there is an oval part of the subsonic area bounded by shocks. These shocks form simple Mach reflections near the slip lines (the contact surfaces emanating from the triple points are only barely discernible here). Configuration B: $\quad \overrightarrow{J_{21} J_{32} J_{34} J_{41}} \quad$ (clockwise motion; Figure 9)

We have $p_{1}=p_{2}=p_{3}=p_{4}$ and

$$
u_{1}=u_{2}>u_{3}=u_{4}, \quad v_{1}=v_{4}<v_{3}=v_{2} .
$$

The solutions have the same symmetry properties as in Configuration A. The structure of the solutions is one of a vortex turning clockwise. The slip lines spiral around its center and the subsonic area has the shape of a four-bladed propeller.

\section{Two neighboring slip lines.}

Configuration C: $\quad \vec{R}_{21} J_{32} J_{34} \vec{R}_{41} \quad$ (Figure 10)

We have $p_{1}>p_{2}=p_{3}=p_{4}$ and

$$
u_{2}-u_{1}=\Phi_{21}, \quad u_{3}=u_{4}=u_{1}, \quad v_{4}-v_{1}=\Phi_{41}, \quad v_{3}=v_{2}=v_{1} .
$$

The solutions are symmetric to $\eta-\xi=v_{1}-u_{1}$. In the region $\xi+\eta>u_{3}+v_{3}$ the solution resembles that of Configuration 2. The slip lines $J_{32}$ and $J_{34}$ meet the sonic circle of the constant state in the third quadrant and continue as almost straight lines so that a quarter of the sonic circle lies in between. 
Configuration D: $\quad \overleftarrow{R}_{21} J_{32} J_{34} \overleftarrow{R}_{41}$

(Figure 11)

We have $p_{1}<p_{2}=p_{3}=p_{4}$ and

$$
u_{1}-u_{2}=\Phi_{21}, \quad u_{3}=u_{4}=u_{1}, \quad v_{1}-v_{4}=\Phi_{41}, \quad v_{3}=v_{2}=v_{1} .
$$

The solutions are symmetric to $\eta-\xi=v_{1}-u_{1}$. The slip lines $J_{32}$ and $J_{34}$ meet the sonic circle of the constant state in the third quadrant and continue slightly bent so that a quarter of the sonic circle lies in between. The $\lambda_{+(-)}$characteristic lines inside $\overleftarrow{R}_{41}$ $\left(\overleftarrow{R}_{21}\right)$ are almost parallel to the contour lines inside the fan and end at the sonic circle. Outside the rarefaction waves the subsonic area is bounded by a circular shock wave.

Configuration E: $\quad \overleftarrow{S}_{21} J_{32} J_{34} \overleftarrow{S}_{41}$

(Figure 12)

We have $p_{1}>p_{2}=p_{3}=p_{4}$ and

$$
u_{2}-u_{1}=\Psi_{21}, \quad u_{3}=u_{4}=u_{1}, \quad v_{4}-v_{1}=\Psi_{41}, \quad v_{3}=v_{2}=v_{1} .
$$

The solutions are symmetric to $\eta-\xi=v_{1}-u_{1}$. The shocks $\overleftarrow{S}_{21}$ and $\overleftarrow{S}_{41}$ intersect the sonic circle of the constant state in the first quadrant and end at the slip lines. Between the slip lines $J_{32}$ and $J_{34}$, which bend inside the subsonic area and end in spirals, the subsonic area is bounded by an oval shock wave. This shock is connected to the shocks $\overleftarrow{S}_{21}$ and $\overleftarrow{S}_{41}$ by two simple Mach reflections.

Configuration F: $\quad \vec{S}_{21} J_{32} J_{34} \vec{S}_{41} \quad$ (Figure 13)

We have $p_{1}<p_{2}=p_{3}=p_{4}$ and the same equations as in Configuration E.

The solutions are symmetric to $\eta-\xi=v_{1}-u_{1}$. The slip lines $J_{32}$ and $J_{34}$ meet the sonic circle of the constant state in the third quadrant and continue as almost straight lines so that a quarter of the sonic circle lies in between. Inside the subsonic area the slip lines bend and end in spirals. The shocks $\vec{S}_{21}$ and $\vec{S}_{41}$ interact like the pair of shocks in Configuration 4.

Configuration G: $\quad \vec{R}_{21} J_{32} J_{34} \overleftarrow{S}_{41}$

(Figure 14)

We have $p_{1}>p_{2}=p_{3}=p_{4}$ and

$$
u_{2}-u_{1}=\Phi_{21}, \quad u_{3}=u_{4}=u_{1}, \quad v_{4}-v_{1}=\Psi_{41}, \quad v_{3}=v_{2}=v_{1} .
$$

The slip lines $J_{32}$ and $J_{34}$ bend after entering the subsonic area and end in a spiral. The rarefaction wave $\vec{R}_{21}$ turns backward in front of the shock $\overleftarrow{S}_{41}$ which ends at the slip line $J_{34}$. The $\lambda_{+}$characteristic lines inside $\vec{R}_{21}$ are almost parallel to the contour lines inside the fan and end at the sonic circle.

Configuration $\mathrm{H}: \quad \overleftarrow{R}_{21} J_{32} J_{34} \vec{S}_{41}$

(Figure 15)

We have $p_{1}<p_{2}=p_{3}=p_{4}$ and

$$
u_{1}-u_{2}=\Phi_{21}, \quad u_{3}=u_{4}=u_{1}, \quad v_{4}-v_{1}=\Psi_{41}, \quad v_{3}=v_{2}=v_{1} .
$$

The slip lines $J_{32}$ and $J_{34}$ bend after entering the subsonic area and end in a spiral. The shock $\vec{S}_{41}$ bends downward before it dissolves inside the rarefaction fan of $\overleftarrow{R}_{21}$ which turns right after crossing the slip line $J_{32}$. The $\lambda_{-}$characteristic lines inside $\overleftarrow{R}_{21}$ are almost parallel to the contour lines inside the fan and end at the sonic circle. 


\section{Two non-neighboring slip lines.}

Configuration I: $\quad J_{21} \vec{R}_{32} J_{34} \vec{R}_{41}$

We have $p_{1}=p_{2}>p_{3}=p_{4}$ and

(Figure 16)

$$
u_{1}=u_{2}=u_{3}=u_{4}, \quad v_{4}-v_{1}=\Phi_{41}, \quad v_{3}-v_{2}=\Phi_{32} .
$$

The slip lines $J_{21}$ and $J_{34}$ which bend and join inside the subsonic area divide the solution into a left and right part. In general the rarefaction waves $\vec{R}_{32}$ and $\vec{R}_{41}$ move with different velocities so that they are shifted against each other. Below these waves the sonic curve is almost circular. Depending on the vertical velocities weak shocks are formed below the rarefaction waves.

Configuration J: $\quad J_{21} \overleftarrow{S}_{32} J_{34} \overleftarrow{S}_{41} \quad$ (Figure 17)

We have $p_{1}=p_{2}>p_{3}=p_{4}$ and

$$
u_{1}=u_{2}=u_{3}=u_{4}, \quad v_{4}-v_{1}=\Psi_{41}, \quad v_{3}-v_{2}=\Psi_{32} .
$$

The slip lines $J_{21}$ and $J_{34}$ separate the solution into a left and right section. Typically, the shocks $\overleftarrow{S}_{32}$ and $\overleftarrow{S}_{41}$ are shifted against each other. Above these shocks the sonic curve is nearly circular. $\overleftarrow{S}_{32}$ and $\overleftarrow{S}_{41}$ are connected by two simple Mach reflections. Their slip lines as well as $J_{21}$ and $J_{34}$ meet in a vortex inside the subsonic area.

Configuration $\mathrm{K}: \quad J_{21} \overleftarrow{S}_{32} J_{34} \vec{R}_{41}$

(Figure 18)

We have $p_{1}=p_{2}>p_{3}=p_{4}$ and

$$
u_{1}=u_{2}=u_{3}=u_{4}, \quad v_{4}-v_{1}=\Phi_{41}, \quad v_{3}-v_{2}=\Psi_{32} .
$$

The slip lines $J_{21}$ and $J_{34}$ which bisect the solution into a left and right portion join in a vortex inside the subsonic area. The shock $\overleftarrow{S}_{32}$ ends at $J_{34}$. Below $\vec{R}_{41}$ the subsonic area is partly bounded by a shock wave.

\section{Conclusions}

The computational results presented here demonstrate the complex phenomenology inherent in the 2D gas dynamic Riemann problem, despite the limitation that each of the four jumps consists of a single elementary wave. The predictions of [19] are close to our results in most cases where this is reasonable to expect. One consistent discrepancy is that predicted shock waves do not exist or that we found unpredicted shock waves and these changes can have further consequences for the overall solution. However, the present work has led to the discovery of many new flowfield patterns; this is especially so for cases involving slip line initial data. For such cases we remark that some of the other cases appear as part of the flowfield pattern, as might be expected.

An important result is that many of the familiar oblique shock wave reflection (OSWR) flowfields appear here. An interesting question is whether or not OSWR is a subset of the 2D Riemann problem, i.e., given a wedge angle and shock wave Mach number [5], can Riemann problem initial data be found so as to construct the given OSWR case? Note that this is nontrivial only because of the restricted set of initial data allowed.

We note here that if the four initial states were separated by two lines not necessarily perpendicular, the number of allowable distinct initial configurations would increase 
to 77 (resp. 75) for isentropic (resp. polytropic) gas. Given the many other possible generalizations, including a change of equation of state, it is easy to see that the $2 \mathrm{D}$ Riemann problem contains many possibilities.

It would appear that this is a very negative result regarding the direct use of the $2 \mathrm{D}$ Riemann problem in constructing an unsplit RCM (for analytic or numerical purposes) or finite difference schemes. However, the set of points in physical space-time involving such complex interactions is of high codimension and it still might be hoped that such schemes are possible. Whether or not the present calculations will prove useful in this endeavor, one can certainly expect that the results here can be used as test or reference cases in the code development process.

\section{ACKNOWLEDGEMENT}

The authors would like to thank Tai-Ping Liu for helpful discussions.

\section{REFERENCES}

1. P. Colella and H. M. Glaz, Efficient solution algorithms for the Riemann problem for real gases, J. Comp. Phys. 59 (1985), 264-289.

2. P. Colella and P. R. Woodward, The piecewise parabolic method (PPM) for gas-dynamical simulations, J. Comp. Phys. 54 (1984), 174-201.

3. R. Courant and K. O. Friedrichs, Supersonic Flow and Shock Waves, Springer-Verlag, New York, 1948.

4. H. M. Glaz, Numerical computations in gas dynamics with high resolution schemes, Shock Tubes and Waves, Proc. Sixteenth Intl. Symp. on Shock Tubes and Waves, H. Grönig, editor, VCH Publishers, 1988, pp. 75-88.

5. H. M. Glaz, Self-similar shock reflection in two space dimensions, Multidimensional Hyperbolic Problems and Computations, The IMA Volumes in Mathematics and its Applications, Vol. 29, J. Glimm and A. J. Majda, editors, Springer-Verlag, New York, 1991, pp. 70-88.

6. H. M. Glaz, P. Colella, I. I. Glass and R. L. Deschambault, A numerical study of oblique shock-wave reflections with experimental comparisons, Proc. R. Soc. Lond. A398 (1985), 117-140.

7. H. M. Glaz, P. A. Walter, I. I. Glass and T. C. J. Hu, Oblique shock wave reflections in SF $:$ a comparison of calculation and experiment, AIAA J. Prog. in Astr. and Aero. 106 (1986), 359-387.

8. J. Glimm, Solutions in the large for nonlinear hyperbolic systems of equations, Comm. Pure Appl. Math. 18 (1965), 697-715.

9. J. Glimm, C. Klingenberg, O. McBryan, B. Plohr, D. Sharp, and S. Yaniv, Front tracking and two-dimensional Riemann problems, Adv. Appl. Math. 6 (1985), 259-290.

10. J. Guckenheimer, Shocks and rarefactions in two space dimensions, Arch. Rational Mech. Anal. 59 (1975), 281-291.

11. W. B. Lindquist, The scalar Riemann problem in two spatial dimensions: piecewise smoothness of solutions and its breakdown, SIAM J. Math. Anal. 17 (1986), 1178-1197.

12. T.-P. Liu, Admissible solutions of hyperbolic conservation laws, Mem. Amer. Math. Soc. \#240 30 (1981).

13. A. Majda, Compressible Fluid Flow and Systems of Conservation Laws in Several Space Variables, Springer-Verlag, 1984.

14. C. W. Schulz-Rinne, Classification of the Riemann problem for two-dimensional gas dynamics, SIAM J. Math. Anal. (to appear).

15. J. A. Smoller, Shock Waves and Reaction-Diffusion Equations, Springer-Verlag, New York, 1982.

16. D. H. Wagner, The Riemann problem in two space dimensions for a single conservation law, SIAM J. Math. Anal. 14 (1983), 534-559.

17. T. Chang (T. Zhang), L. Hsiao (L. Xiao), The Riemann Problem and Interaction of Waves in Gas Dynamics, Longman Scientific and Technical (Pitman Monographs No. 41), Essex, 1989.

18. T. Zhang, Y. Zheng, Two-dimensional Riemann problem for a scalar conservation law, Trans. Amer. Math. Soc. 312 (1989), 589-619. 
19. T. Zhang, Y. Zheng, Conjecture on the structure of solutions of the Riemann problem for twodimensional gas dynamic systems, SIAM J. Math. Anal. 21 (1990), 593-630. 